\title{
APPLICATION OF TEXTURE SCHEMES IN ARCHITECTURAL DRAWING AN ANALYTICAL STUDY -FIRST YEAR STUDENTS' DRAFTS IN DEPARTMENT OF ARCHITECTURE IN UNIVERSITY OF DUHOK
}

\author{
ARMANGE ISMAEL BARAKAT \\ Dept. of Architecture, University of Duhok, Kurdistan Region-Iraq
}

\begin{abstract}
The determination of schemes of texture considered an essential need to get a full understanding of the research problem which represented by the lack of clarity about the role of texture characteristics which adopted in forming the texture schemes in architectural drawing. In architectural design the texture strengthens our understanding of quality of form, in addition to that texture can help students acquire unique visual features in their drafts. Accordingly, this paper focuses on studying the texture characteristics in architecture and how the students can use these characteristics to create their textures. So this paper aims to explore the possibilities of texture schemes that used in architectural drawing. The first year students' drafts in the department of architecture have been analyzed using geometrical methods to define the strategies for putting texture schemes of these drafts. The results showed that the students adopted a set of characteristics of texture more than others in their schemes. The characteristics have been used on two levels: the first one is the micro level related to texture motifs, which work to generate the texture structure that represents the macro level of the scheme. This paper provides a vision about the importance of texture characteristics such as motifs shape, motifs scale, the natural pattern of motifs and directionality of motifs, at the first level and at the second level the types of texture, the roughness of texture, texture reflection and texture relation that have been used. The focus has been on forming the texture wheel, which in turn gives the students ideas about other characteristics of texture and helps to use them in their work.
\end{abstract}

KEY WORDS: Architecture, Design, Texture Scheme, Texture Characteristics, Texture Structure, Texture Motifs.

\section{INTRODUCTION}

$\mathbf{T}$ he term of texture in all visual arts in many cases comes as a stimulant factor that gives different impressions and has several effects, such as aesthetic effects which appear on the surfaces of the final product. Designers, through ages, worked to take a benefit from the flexibility of texture as a design element and influential item to add visual interest and visual variety in their works. The texture consists of a set of visual characteristics that are organized according to the principles of design, which work as composition. The emphasis on one or more of these characteristics in different situations works to achieve richness in design in general and in architectural drawing in specially. Thus appears a need for studies that should discuss these issues in detail in order to realize their potentials in enriching the design, the students of architecture are studying the design elements and the texture is one of them. Accordingly, the basic theme of the current research was chosen to identify the nature of visual characteristics of texture in architecture and other visual arts and how can be applied in the students' drafts.

\section{LITERATURE REVIEW}

This study tries to review a group of studies and proposals that addressed texture in visual arts, particularly architecture, some of these studies dealt with texture in general way and others had analyzed the characteristics of texture.

Graves study analyzes and explains the design elements in certain situations where each element has a group of inspirational cases and a range of potentials that assist to enrich the design. It is worth mentioning that the texture is considered as an important design element as size, shape, and color in visual arts. On the other hand, the study confirms that modern designers can be accredited for recognizing this importance, and for reviving the use of textural variety because of its aesthetic value in painting, architecture, interior design, sculpture, and industrial design, with the introduction of new materials. It has been demonstrated in this study that all surfaces in visual arts are variations of one of the four basic textures -rough matt, rough glossy, smooth matt, smooth glossy. In addition to the actual textures, 
there are simulated or artificial textures. Simulated textures are mechanical or hand-made (Graves, 1951, p. 222).

While Ching defines the texture as surface characteristics of a form; it is a "texture affects both the tactile and light reflective qualities of a form's surfaces"( Ching,1979, P.50).On the other issues, the study states that the form can be articulated by differentiating planes with a change in material, color, texture, or pattern. Perception of the shape, size, scale, proportion, and visual weight of plane is influenced by its surface properties as well as by its visual context. Ching added that both texture and color together affect the visual weight and scale of plane and the degree to which it absorbs or reflects light and sound (Ching, 1979, pp.81, 88-89).

And Shirzad defines texture as the exterior appearance to the natural or industrial things that we see or feel by the hand, and that include the difference in smoothness, roughness, hardness, and transparency, and it also includes the decoration, inscriptions and sculptures. In large formations or large designs that could be seen from a distance, the large elements like a singular house alone within residential compounds can produce a special influential tactile on the overall composition of the design area (Shirzad, 1985, P. 143).

Whereas Rasmussen talks about the textural effects; he confirms that the observer must experience the texture effects by him/ herself and they have to perceive the importance of texture effects. The study shows the texture effects in other fields, like Basketry, Pottery with their geometrical patterns, and the contrast between their textures (rough, smooth). (Rasmussen, 2008, p.160-180).

Guo et al. carried out a study to determine five important characteristics affecting the perception of visual complexity of texture: regularity, roughness, directionality, density, and understandability. The study confirmed that understanding of visual perception of textures takes various kinds of applications from computer science to arts, architecture, and the design of product surfaces, packages, carpets, and wallpapers. For instance, understanding the visual perception of textures is useful in the process of image retrieval and it helps artists or architects choose a texture for their work or a product that motivates a particular emotion. (Guo et al, 2012, p. 306).
The findings and the main conclusion of those studies can be summarized as follows:

Texture is an important design element, and it is one of the visual properties of form as demonstrated by these studies. In other words, these studies addressed the descriptive characteristics of texture, like rough, smooth, glossy and matt. However, we think that the above mentioned studies can be useful to current research as their results and approaches can be used as important indicators for discussion. The studies have not shown the mechanism of using the characteristics of texture in architectural design and architectural drawing, that a student can count to use these characteristics in the drafts. Therefore, the problem of the research was identified on the level of clarity of the adopted mechanism for put the texture scheme in architecture and architectural drawing particularly.to solve this problem the following hypothesis was adopted: the texture scheme is based on texture characteristics and relations between these characteristics which work collectively to form the texture scheme. Also, the objective of the study was established: to define the potentials of the texture characteristics that used in forming the texture scheme and using it in architectural drawing.

\section{CHARACTERISTICS OF TEXTURE}

\subsection{Texture types}

Texture in visual arts can be classified into four types as shown in (figure 1). They are:

3.1.1 Actual texture: this type is considered the "real thing"; it is the way the surface of an object looks and feels. Generally, the emphasis is on the way it feels to the touch. This texture can also give a preliminary idea of the feel by viewing the object (Ocvirk, 2006, p.131).

3.1.2 Simulated texture: This type of texture is a surface character that looks real but, in fact, it is not said to be a simulated texture (Ocvirk, 2006, p.131).

3.1.3 Abstract texture: This kind of texture usually displays some hint of the original texture but it has been modified to suit the artist's particular needs (Ocvirk, 2006, p.131).

3.1.4 Invented texture: This is a texture without precedent; it does not simulate nor is abstracted from reality; it is purely the creation of the artist's imagination. A created texture which only sources from the imagination of the artist is called an invented texture. (Ocvirk, 2006, p.131). 


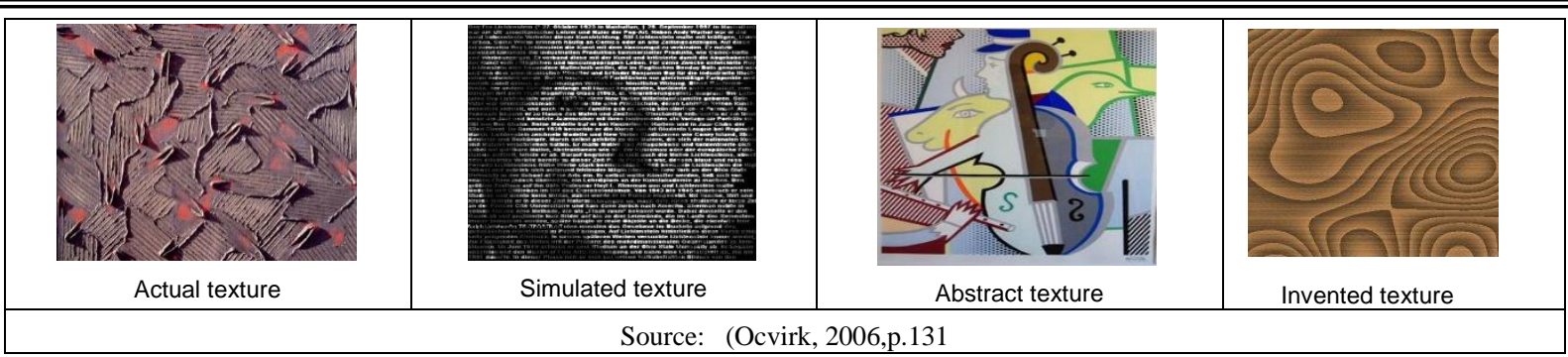

Fig. (1): Texture Types

\subsection{Texture classifications}

Texture can also be classified according to perception of texture; where we experience the texture with two of senses: touch and vision. Therefore, texture may be classified into two important categories: visual texture and tactile texture. An appropriate texture adds richness to the design (Wong, 1993, p.67-68), and as follows: 3.2.1Visual texture refers to the illusion of the surface texture. It is concerned with how a tactile texture looks (Steed, Stevenson, 2012, p.114). In other words, a visual texture is" precisely twodimensional. It is the kind of term that is seen by the eye, although it also may evoke tactile (touchable) sensations" (Wong, 1993, p. 68). Three kinds of visual textures can be distinguished, which are:

- Decorative texture. This decorates a surface, and remains subordinate to shape.

- Spontaneous texture. This texture does not decorate a surface, but it is part of the process of optical creation.

- Mechanical texture. A typical example of this kind of texture is the photographic grain or screen pattern we often find in printing as shown in figure(2)

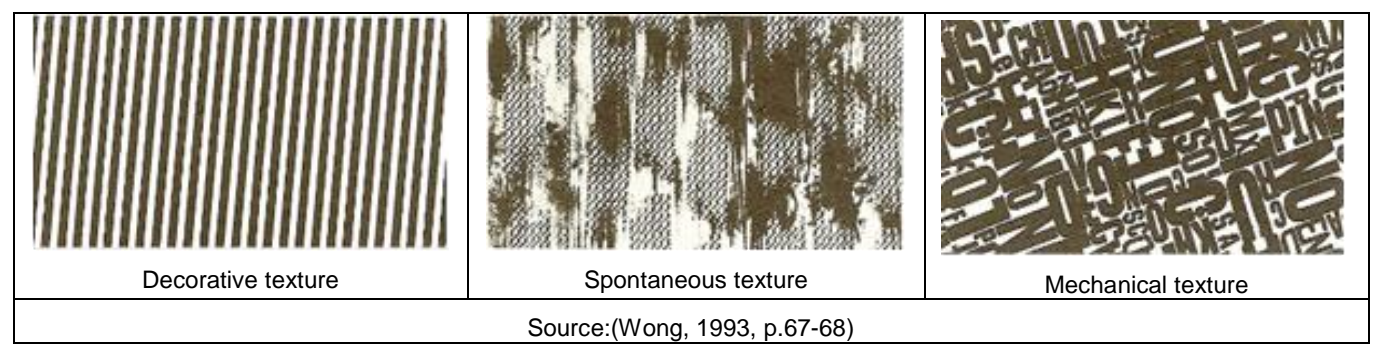

Fig. (2): three kinds of visual textures

3.2.2. Tactile texture. The term of tactile means touch. Tactile texture refers to the feel of a surface.

The actual surface texture needs to be felt or seen with light across its surface to make the texture visible (Steed, 2012, p.114). In other words, tactile texture is a classification of texture that is not only visible to the eye but it can be felt with the hand. Tactile texture rises above the surface of a two-dimensional design and approaches a three-dimensional relief form (Wong, 1993, p.64). As shown in figure (3)

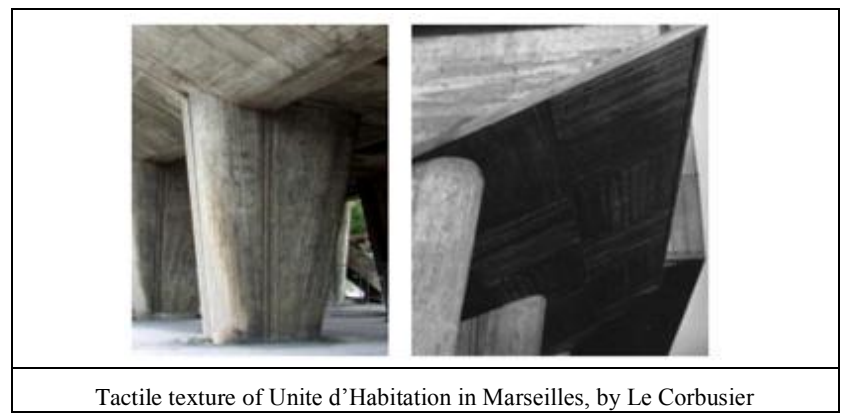

Fig. (3): tactile texture 


\subsection{Texture Motifs}

The term motif refers to any element in design (Dictionary). It typically applies to a repeated element. However, it can be used for non-repeated elements as well. In addition to that, motif could be defined as a decorative design or decorative pattern. Motifs are affected by a set of factors that are motifs shape, spacing and scale of motifs, repeat type, directionality and orientation of motifs, color, and style of motifs (Kight, 2011, p. 9-13).

\subsubsection{Shape of Motifs.}

There are four basic styles or shapes of motifs namely; Geometric, Realistic, Stylized, and Abstract (Koester, 1993, p.3), as shown in figure (4).

- Geometric motifs such as stripes and plaids (Koester, 1993,p.3), and motifs shape could be polka dots , diamonds, triangles, lines, squares, circles, etc. or any geometric shapes, in addition to geometric patterns that can be simple or complex, regimented or random, straight or curvy or anywhere in between (Kight, 2011, p.15).

- Realistic motifs. Realistic motifs are imitations or repeats of natural or manmade objects" (Koester, 1993, p.3) such as organic shapes, flowers, etc. Kight (2011) considers this category with more gravitational pull than geometric in design (Kight, 2011, p.17).

- Stylized motifs. These are simplified variations of natural or man-made objects that are no longer recognizable (Koester, 1993, p.3).

- Abstract motifs. They are combinations of color, size, and shape without relationship to natural or man-made objects (Koester, 1993, p.3).

And koester proposed that realistic, stylized, and abstract motifs may be easier or more difficult to use depending on three important factors, which are: first is the size of the motif, the second is the contrast between the motif and the background, and the third one is the direction of motifs (multiple-direction or one-way) (Ibid.).

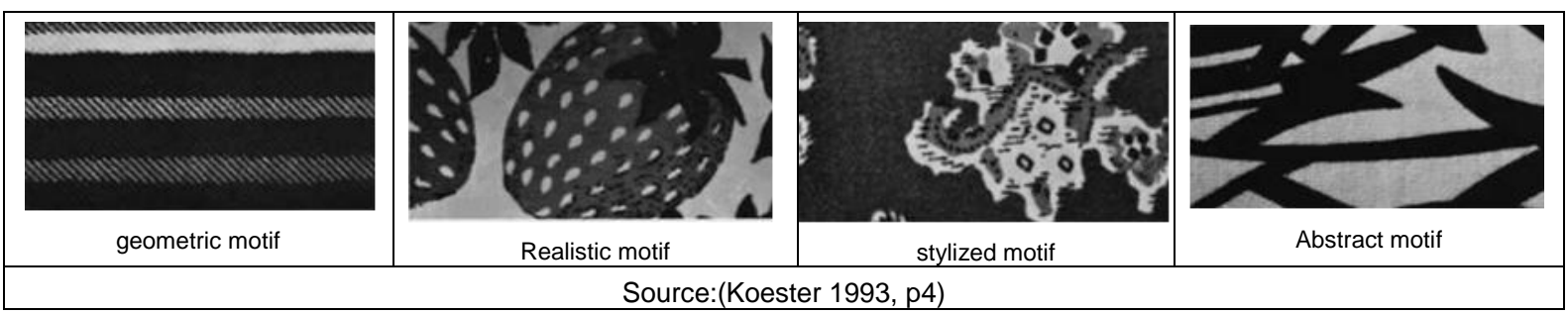

Fig. (3): types of texture motifs

\subsubsection{Spacing and scale of motifs.}

For the spacing, motifs can be packed together, showing little to no background, or spaced showing lots of background. Designs with altering packed and spaced areas are called open and closed. They might flow together via continuous, connecting elements or appear to float on their own. These are aesthetic choices that do not have strong correlations to a texture's end uses (Bell, 2004, p.69); this is shown in figure (4).

Scale is very important in relation to the end uses of texture. Also, motifs can range from minuscule (pin dots) to the super large. Though, scale is somewhat relative depending on application. Thus, there are three relative types of scales as follows :(Kight, 2011, p.25).See figure (4).

- Small scale motifs =: (10) $\mathrm{mm}^{2}$ and under

- Medium scale motifs = in between $(50-100) \mathrm{mm}^{2}$ - Large scale motifs: Greater than (100) $\mathrm{mm}^{2}$

This classification of shape and scales of motifs is widely used in textile design. The current study will depend on this classification in analyzing the shape and scale of motifs in first stage students' drafts in architectural department. 


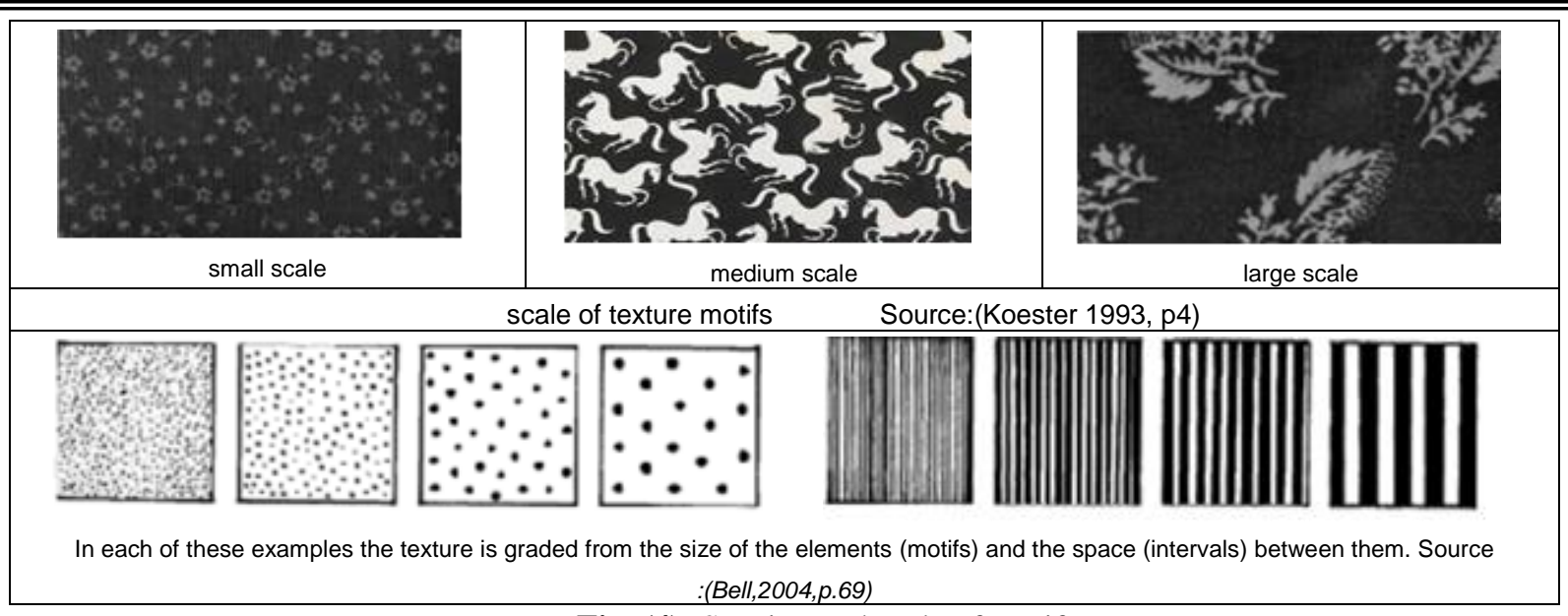

Fig. (4): Spacing and scale of motifs

\subsubsection{Natural patterns of motifs.}

Texture and pattern are related. When we look closely at an object such as a tree, we can see the pattern of leaves that make its surface. When we back away, we lose awareness of the leaves and instead we notice the texture that the leaves make on the tree. Also, most of us see patterns as a collection of forms, lines, symbols, tones and colors. A pattern is used extensively in visual art design; it is most often used in an aesthetic way to appeal the senses to be good-looking, attractive, radical, or visionary. Therefore, it is a sensorial experience for the observer or user, and designers must use their knowledge and skill to make patterns serve this purpose. A pattern has its roots in nature and designers of all descriptions often use nature as their source of inspiration (Steed, Stevenson, 2012, pp.116-123).

There are five natural patterns used in all design fields (as shown in figure 2-5).They are as follows:

- Branching pattern. These are seen regularly in design, where rooted motifs spread across the surface or are constructed to form the whole. This pattern looks like a tree growth from their roots to their outer branches (Steed, Stevenson, 2012, p. 123).

- Meanders pattern. The meander is a wandering line like a long winding river. It can be smooth and sleek and single or there can be lots of them together, giving an impression of movement (Ibid).

- Bubbles pattern. This pattern is similar to "the way a corn on the cob has bubbles sticking side by side or the way in which soap bubbles. Bubble formations create texture as well as pattern" (Ibid).

- Explosions pattern. The explosion pattern is used extensively in texture design and is often recognized as simple spotted motifs or freefloating forms across the design (Ibid).

- Spirals pattern. Spirals are one of the most culturally prominent symbols and they reflect different meanings in nearly every culture. The spiral represented eternity within Celtic culture, for example. Spirals can be found in many natural phenomena such as shells, pine cones and fern leaves (Ibid).

These five pattern structures have been used in many of design structures and surfaces, from architecture to textiles and graphics.

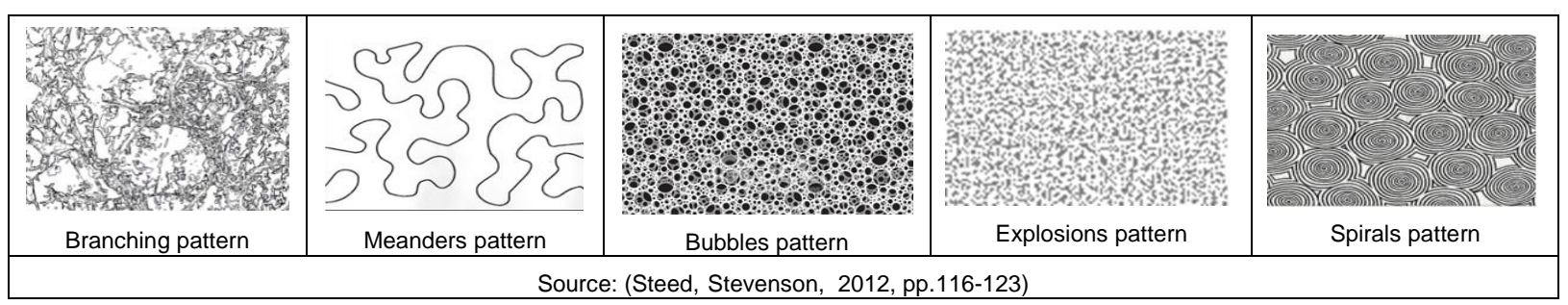

Fig. (5): natural pattern of motifs of texture 


\subsubsection{Directionality of motifs}

Direction in texture pattern design refers to the number of the ways in which motifs or units can be rotated but they keep the same looking. It is both an aesthetic and functional choice. Directionality in texture pattern can be one- way, two-way, four - way or multi- ways (Irawan, 2006, p.1) and as follows:

- One way direction pattern. It is the direction pattern in which motifs can only be turned one way. This is the most natural direction. In the one way pattern all pieces must be oriented in the same way as shown in figure (6) (Kight, 2011, p.10).

- Two ways direction pattern. According to this pattern motifs are turned either right side up or upside down or horizontal and vertical at the same time, as shown in figure (6) (Ibid).

- Four ways or multi-ways direction pattern. In this pattern motifs are either turned or oriented; for example at $0^{\circ}, 90^{\circ}, 180^{\circ}$ or $270^{\circ}$. There is also another choice for motifs position, where it could be with no direction. In such a case, it is called tossed, as shown in figure (6) (Ibid).

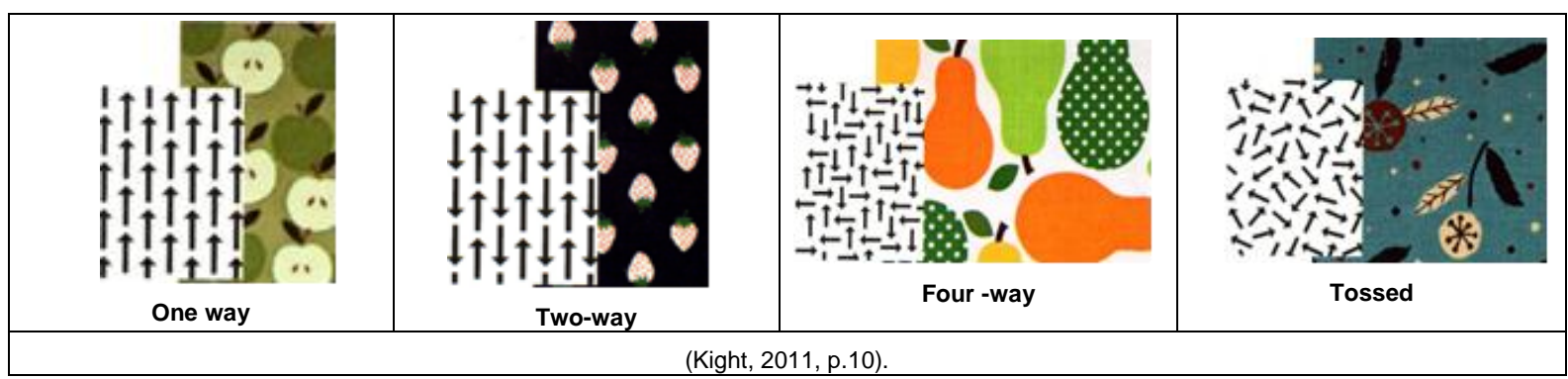

Fig. (6): Directionality

\subsection{The structure of texture}

Structures of texture come in many different forms, as mentioned earlier. Some are soft, but others are hard or rough; our environment contains and is surrounded by utilitarian, functional and it is also highly decorative and patterned structures of texture. Depending on the type of texture that the designers are creating, the designer will need different types of visual information. (Steed, Stevenson, 2012, p. 116). Also, the appearance of a surface texture depends on how it reflects light. Every surface displays a pattern of light and dark values. From the pattern of light and dark values, the user or the viewer can make a judgment about the texture of a surface or an object even if he/she cannot touch it. And this presents the relation between a texture and its value, and gives the structure of texture descriptive values like smooth texture, rough texture, matt or glossy texture and so on. In this way the roughness or smoothness of a texture can be determined by looking at its light and dark values. A rough surface reflects light unevenly. It shows irregular patterns of light and shadow. A smooth texture reflects light evenly (Ragans, 2015, p.174). Examples on matt surfaces can come under stone, concert, and natural wood. A glossy surface is the opposite of a matte surface.
A glossy surface is a surface that reflects so much bright light that seems to glow. Glossy surfaces also have highlights. Some surfaces reflect bright sunlight. The glass, a polished brass, and the surface of some marble are examples of glossy surfaces. Matt and glossy surfaces can be rough or smooth. Sand paper is matte rough, Aluminum foil is glossy and smooth until it gets crumpled up; then it becomes glossy and rough (Ragans, 2015, p. 174-175).

\subsection{Texture and Emotion}

We can manipulate textural qualities and use materials in a contradictory fashion to elicit a mix of emotional responses. A texture manifests itself through its tactile and its visual qualities to convey a range of messages and emotions. Different textures evoke different types of responses. Some surfaces invite touch, while others are repellent and so are the textures that suggest those surfaces. Most textures have a naturalistic quality where they repeat a motif in a random way (Steed, Stevenson, 2012, pp.112-). A texture can create optical illusions that can make the form look larger, smaller, heavier or lighter. This is done as follows: smooth texture emphasizes minor form irregularities. Therefore, it is attractive on the well-proportioned forms; rough textures add size 
to the form, and dull or matt textures absorb light. It usually makes a form look smaller. A glossy texture makes the form appear larger (Hackler, 1988, p.8).

\subsection{Texture and materials}

there are three trends in architecture in terms of the use of tactile texture: "the use of rough textures, the use of smooth textures and the use of both of these textures to create contrast in all of them. However, the texture appears as the outcome of different materials. It must be noted that every material should be used in accordance with its character. You cannot use steel as brick or stone as steel. You should produce the form that the material naturally fits to steel and glass have sharp and smooth surfaces" (Roth, 2012, p.83). In this way, the current research discusses some material's texture potentials as follows:

Stone, brick, concrete, and tiles are commonly used, where the stone has been used for many thousands of years in the construction of buildings. As man developed tools, he/she began to manipulate and fashion the stones that he found around him as society developed. (Gagg, 2012, p.12-15). This is shown in figure (7a).

As for the texture of brick potentials, which are explained in Baker House, in addition to a visual rhythm, Alvar Alto has used rough clinker bricks to be able to give the facades of building a tactile texture. Moreover, he had the bricks laid in a random pattern to add visual texture (Roth, 2012, p.83). This is shown in figure (7b).

As for the concrete texture, "concrete has very much potential to create a tactile and a visual texture because it takes the shape of the molding form to which it was poured into and it also takes the texture of the material of that molding form. In addition to that, joints appear between the successive pours of concrete that mark different pours of concrete. Architects can give special attention to those joints and use them to create a texture. In Salk Institute (at La Jolla) by Louis Kahn for example, the joints of concrete are used to create texture" as shown in figure (7c).

Concerning the tiles; they have been used since roman times to create durable and ornamental surfaces, both inside and outside buildings. However, the ability to break down complex pattern and designs into smaller, manageable units or to apply thinner, cheaper layers of often expensive onto a module (easily handled by a person) has ensured that tiles in whatever material they are made- have remained one of the key materials in applying color and texture to interior and exterior surfaces (Gagg, 2012, p.126-127). This is shown in figure

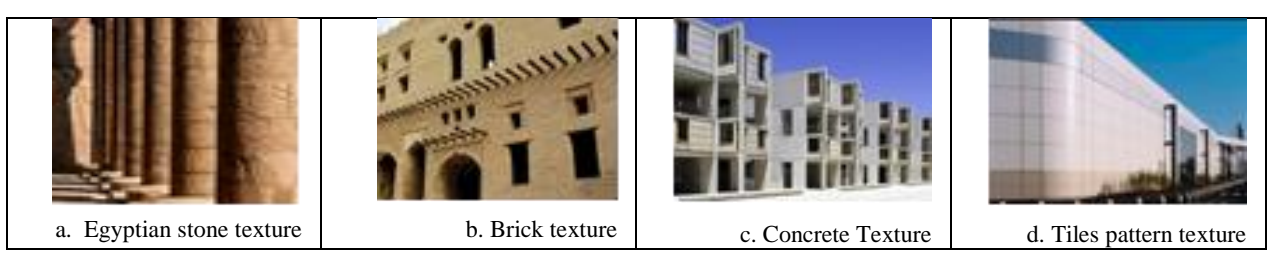

Fig. (7): Texture and Materials

\section{A THEORETICAL FRAME WORK OF DESIGN TEXTURE}

Through reviewing the previous studies, it become clear that the texture characteristics have main variables and sub-variables. In other words the characteristics have been used on two levels: the first one is the micro level related to the variables of texture motifs, as motifs shape, motifs scale, the natural pattern of motifs and directionality of motifs, and at the second level comes the variables of texture structure, which includes the types of texture, the roughness of texture, texture reflection and texture relation that have been used. That are forming the important base for the case study of the research. As shown in the table (1) below. 
Table (1): Variables to measure the samples

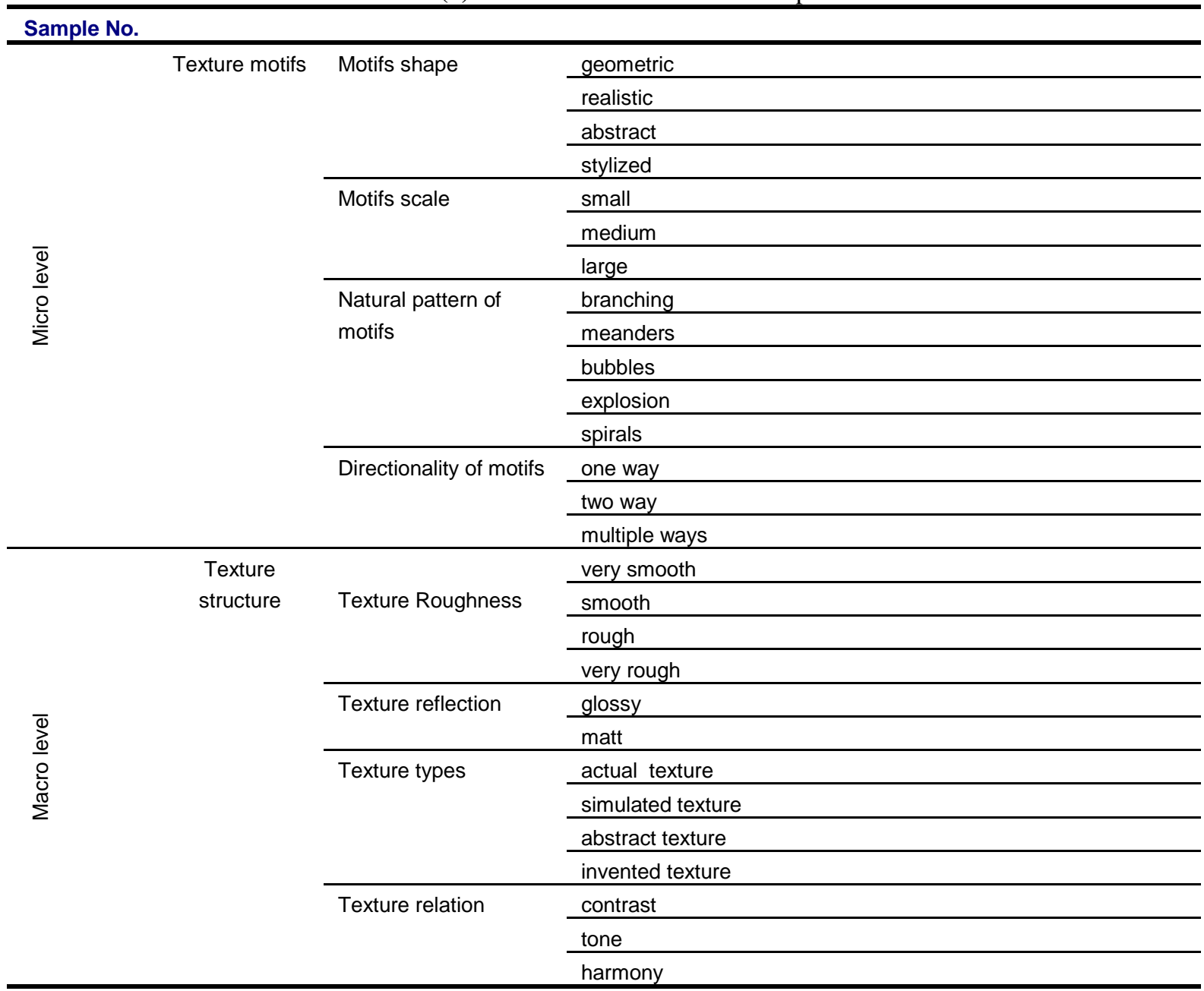

\section{THE CASE STUDY}

In this part of the research, the first year students' drafts in the department of architecture were selected; they were supervised by the subject matter experts, professors well experienced in dealing with the students at this level. The focus was on developing the process of thinking and skills in forming the texture scheme and adopting it in architectural drawing. The basic scheme for all students was selected, which serves the purpose of a unified texture. 20 drafts were selected to be analyzed using the geometrical method to measure the items and the variables of theoretical framework. The required measurement cases of the variables have been summarized in two levels as shown in table (1), as follows:
At the first level comes the analysis of texture motifs, which includes analysis of four sub variables:

1.1 Motifs shape analysis, in terms of being geometric, realistic, stylized, and abstract.

1.2 Motifs scale analysis, by being small, medium, or large scale.

1.3 Natural pattern of motifs analysis, by being branching, meanders, bubbles, explosions, and spirals.

1.4 Directionality of motifs analysis; this analysis measures the variety of direction of motifs in one repetition, which are one way, two ways, or multiple ways.

At the second level comes the analysis of texture structure, which includes analysis of four sub variables:

2.1Texture roughness analysis, in terms of being very smooth, smooth, rough, very rough. 
2.2 Texture Reflection analysis, by being glossy, matt.

2.3Texture types analysis, by being actual, simulated, abstract, and invented texture.

2.4 texture relation analysis, this analysis measures the type of relation between different textures in the same scheme, by being contrast, ton, and harmony.

We have put the geometrical measurement table (1-1) for the sample number 1 in this part as following. (See Appendix (A) for the other analysis forms of the rest samples)

Table (1): Variables to measure the sample (1)

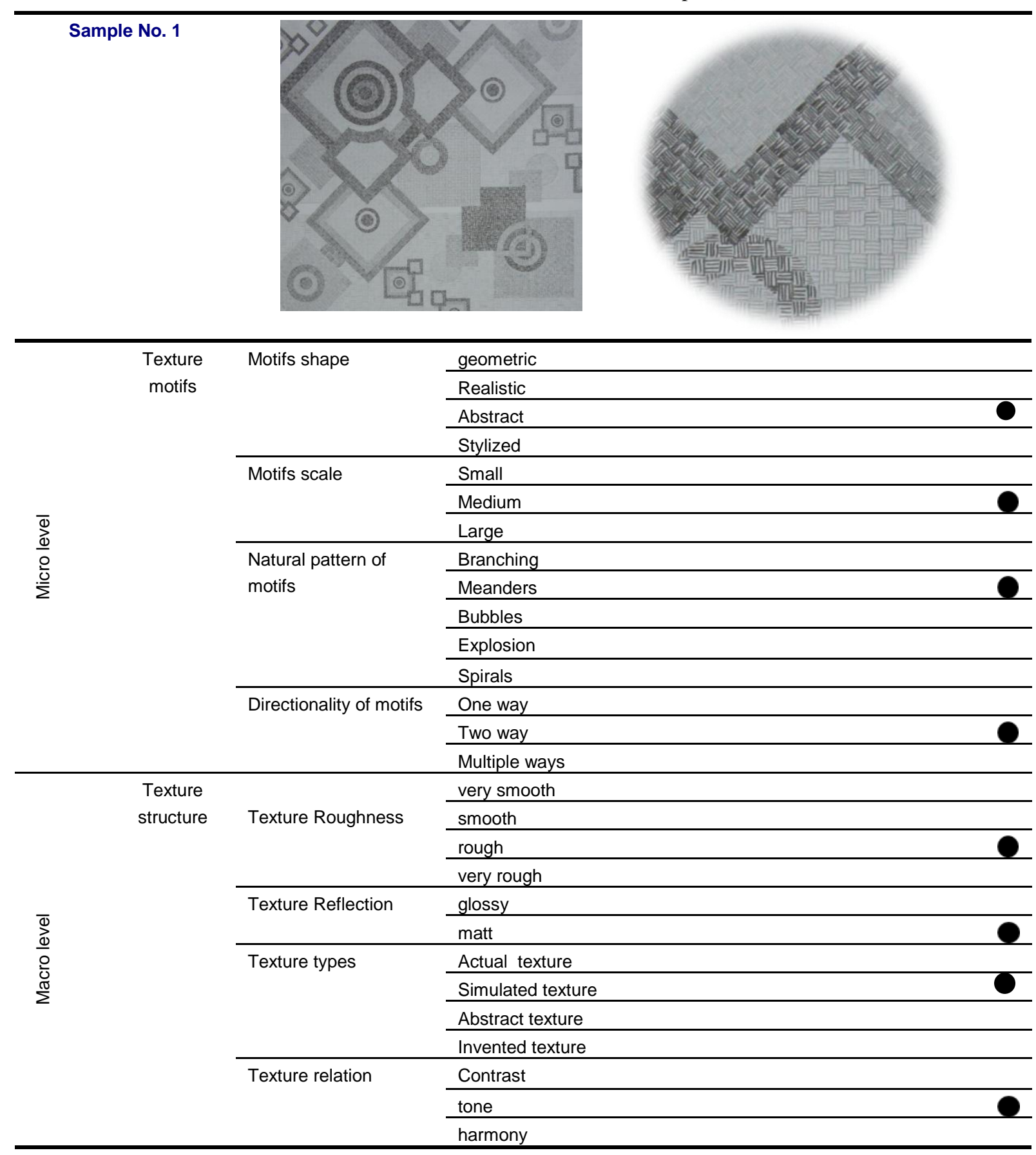




\section{. RESULTS OF THE CASE STUDY}

The results of the case study samples come from the geometrical analysis of the students draft samples which are supported by a statistical analysis. It includes the discussion of the texture characteristics results and its variables.

At the micro level, the percentages of the variables were as follows: it was shown that $95 \%$ of the samples used the abstract type of motifs shape and 5\% used the stylized type. The rest types (geometric, realistic) did not used. The result of motif scale was shown that the highest result was in the variable of medium scale and that percentage $70 \%$. Then came the percentage of small scale was $20 \%$, while the lowest result was the large scale with $10 \%$. The percentages of the variables of natural pattern of motifs were as follows: the greater ratio was in the variable of meanders pattern and that ratio was $90 \%$, while the lower result was the bubbles pattern with $10 \%$. While the rest types of natural pattern of motifs (Branching, Explosion, Spirals) didn't used. The results of the variables of the directionality of motifs represented that the highest percentage was the multiple ways and that percentage was $90 \%$ and the lowest one was the two way with $10 \%$, and the variable of one way did not used. At the macro level, the percentages of the variables were as follows: it was shown that $70 \%$ of drafts samples used the rough texture, while $20 \%$ of them used the smooth texture, while $10 \%$ of them used the very rough texture. Result concerning the texture reflection showed that $100 \%$ of samples used the matt texture. Result concerning the texture types showed that the greater percentage was used the abstract texture and that percentage was $80 \%$, and the lower used was the simulated texture with $20 \%$, while other types of texture (Actual, Invented) did not used. The result of texture relation represented that $100 \%$ of samples used the tone relation.

\section{CONCLUSIONS}

The study has come up with the below conclusions in accordance to the discussion of the results obtained from the geometrical method
6

adopted in the analytical study. Accordingly, the concept of texture characteristics of students' drafts are based on a set of variables which can be characterized to be a scheme, however The results showed that there was disparity in achieving rates of these items within the texture due to that the students adopted a set of characteristics of texture more than others in their drafts, as follows:

- there is a lack of diversity in using the variables of motifs shape, where the abstract shape was the highest rate of use, and the lowest one was the stylized shape, while the rest shapes (geometric, realistic) did not used.

- there is disparity of use within the variables of motifs scale, where the highest rate was in the variable of medium scale, then came the small scale at the second rate of use, while the lowest rate was the large scale.

- Also, there is a lack of diversity in using the variables of natural pattern of motifs, where the greater ratio was in the variable of meanders pattern while the lower result was the bubbles pattern. While the rest types of natural pattern of motifs (Branching, Explosion, Spirals) didn't used.

-The results of the variables of the directionality of motifs represented that the highest percentage was the multiple ways which gave a good variety in texture scheme within this item.

- The major structure of texture that used in students' drafts was rough matt texture, which considers a good strategy in texture scheme for architecture drawing.

- Result concerning the texture types showed that there was a lack in using different types of texture, precisely the invented texture did not used, where this type gives students an opportunity to create their texture.

-The drafts have been dependent on the tone relation as a main relation. And the rest relations as contrast and harmony, came as a result form using the tone relation in whole texture composition.

-to sum up, the forming of the texture wheel as shown below gives the students more understand and ideas about other characteristics of texture and helps them to use these items in their drafts. 


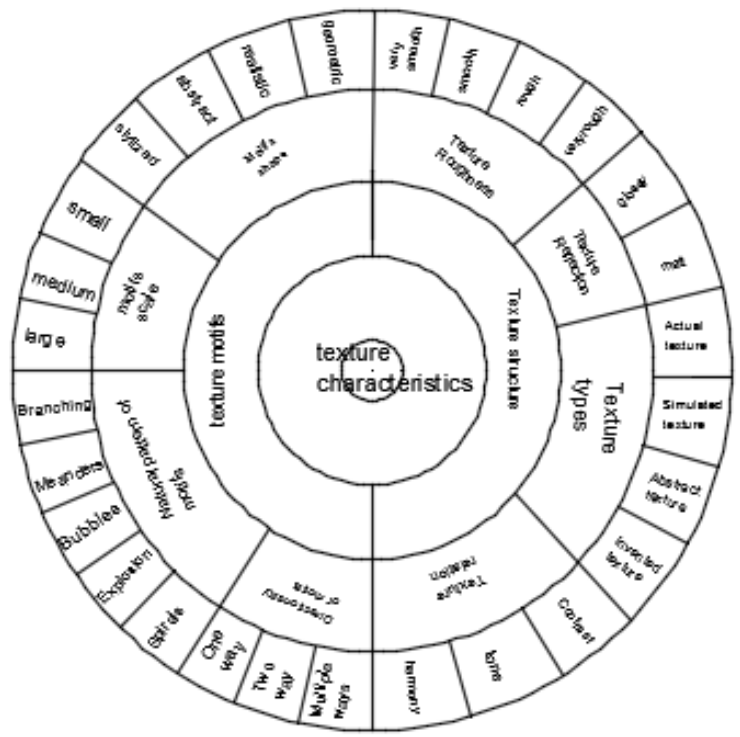

Fig. (8): texture wheel Prepared by researcher

\section{REFERENCES}

1. Bell, Simon. (2004). Elements of Visual Design in the Landscape (Second Edition). London and New York: Spon Press is an imprint of the Taylor \& Francis Group.

2. Ching, Francis. (1979). architecture: form, space \& order. New York: van Nostrand Reinhold Company, INC.

3. Gagg, Russel. (2012). Basics introduction architecture: texture +materials. USA, ava academia: AVA publishing SA.

4. Gagg, Russel. (2012). Basics introduction architecture: texture +materials. USA, ava academia: AVA publishing SA

5. Graves, Maitland. (1951). the art of color and design (second edition). McGraw: hill book company, INC.

6. Guo, Xiaoying. Asano, Chie Muraki. Asano, Akira. Kurita, Takio. And LI, Liang. (2012). Analysis of Texture Characteristics Associated with Visual Complexity Perception (pp.306-314). Japan: published by The Japan Society of Applied Physics.

7. Hackler, Nadine. (1988).4-H clothing Selection. Florida cooperative extension service institute of food and agricultural sciences, university of Florida: IFSA

8. Irawan, Piti. \& Stephen Marschne R. (2006). A Simple, Accurate Texture Model for Woven Cotton Cloth. Cornell University Program of
Computer Graphics: Technical Report PCG-0601

9. Kight, Kimberly. (2011). a Field Guide to Fabric Design: Design, Print \&Sell Your Own Fabric. Traditional \&Digital Techniques for quality, home Dec\& apparel. America: Stash Book, an Imprint of C\&T Publishing, Inc.

10. Koester, Ardis W. (1993). Extension textiles and clothing specialist. USA, Oregon State University: Oregon state university extension service.

11. Lanes in Mosul City. University of Mosul, unpublished master's research.

12. Ragans, Rosalind. (2015). Art Talk: texture. Retrieved march 3, 2015from http://www.glencoe.com/sec/art/art_talk/index.p $\mathrm{hp} / \mathrm{al}$

13. Rasmussen, Steen Eider. (2008). Experiencing Architecture: Translation Mohammed bin Hussein Al Ibrahim. Beirut: Dar Gabes for printing, publishing and distribution.

14. Roth, Leland M. (2012). Understanding Architecture: Its Elements, History and Meaning (Second Edition Editions). United States of America: Westview press, A Member of The Peruses Books Group.

15. shirzad, Shireen Ehsan. (1985). Principles in art and architecture. Baghdad: Printed in the Arab House.

16. Wong, Wucius. (1993). Principles of Form and Design (1st Edition). New York: John Wiley \& Sons,

INC. 